\title{
The association between functional connectivity and cognition in older adults
}

\author{
Sarah Khasawinah ${ }^{1,2 *}$, Yi-Fang Chuang ${ }^{3}$, Brian Caffo ${ }^{4}$, Kirk I Erickson ${ }^{5}$, Arthur F Kramer ${ }^{6,7}$ and Michelle C Carlson ${ }^{1,8}$ \\ ${ }^{1}$ Department of Mental Health, Bloomberg School of Public Health, Johns Hopkins University, Baltimore, Maryland, USA \\ ${ }^{2}$ Research Foundation for Mental Hygiene, Inc., New York, New York, USA \\ ${ }^{3}$ National Yang Ming University, Taipei, Taiwan \\ ${ }^{4}$ Department of Biostatistics, Bloomberg School of Public Health, Johns Hopkins University \\ ${ }^{5}$ Department of Psychology, University of Pittsburgh, Pennsylvania, USA \\ ${ }^{6}$ Beckman Institute, University of Illinois, Urbana-Champaigne, USA \\ ${ }^{7}$ Department of Psychology, Northeastern University, Boston, Massachusetts, USA \\ ${ }^{8}$ Center on Aging and Health, Johns Hopkins University
}

\begin{abstract}
In this cross-sectional study of older adults, we investigated two fundamental brain networks whose coupling is known to be associated with cognitive aging and dementia risk, the Task Negative Network and the Task Positive Network. We investigated connectivity using data from functional magnetic resonance brain imaging (fMRI) with a working memory task in the Brain Health Study (BHS), a study nested within the Baltimore Experience Corps Trial. BHS participants $(n=85)$ were socio-demographically diverse community-dwelling adults over the age of 60 . We used a priori derived regions of interest from the literature, and developed a linear regression-based method using Pearson's correlations as measures of network connectivity and scores on neuropsychological tests, administered outside of the scanner. The cognitive domains assessed were executive functions (task switching and working memory) and memory (episodic memory), which are known to be associated with dementia risk. We found that memory was both positively and negatively associated with inter-network connectivity, depending on the sub-network, while executive functions were negatively associated with inter-network connectivity. Sex modified these results, indicating underlying biological differences in the associations between brain network connectivity and cognitive functions in men and women. These results provide support for the potential utility of this paradigm when applied to the analysis of fMRI data to detect pre-clinical changes in cognitive functions.
\end{abstract}

\begin{abstract}
Abbreviations: AD: Alzheimer's disease, BHS: Brain Health Study, BECT: Baltimore Experience Corps Trial, CSF: cerebrospinal fluid, dMFC: Dorsal medial prefrontal cortex, DST: Digit Span Test, EC: Experience Corps, EF: Executive Function, IPL: Inferior parietal lobule, LTC: Lateral temporal cortex, MNI: Montreal Neurological Institute, TMT: Trail Making Test, TNN: Task Negative Network, TPN: Task Positive Network, PCC: Posterior cingulated, PHC: Parahippocampal cortex, PFC: Prefrontal Cortex, RAVLT: Rey Auditory Verbal Learning Test, Rsp: Retrospenial cortex
\end{abstract}

\section{Introduction}

Aging populations are changing the demographics of developed nations across the world, presenting increased risk of cognitive decline as a global public health epidemic. In this investigation, we sought to add to the literature of neuronal markers of cognitive differences. We sought to detect heterogeneity in cognitive aging that may signal risk and resilience as measured by the dynamic relationships between a task negative network, aptly known as the default mode network, and a task positive network. Brain networks are collections of regions that are functionally connected and likely work together to produce complex cognition and behavior. The synchronous activations of brain networks indicate healthy functioning, and interruptions may hold a key to dysfunction.
The Task Positive Network (TPN) represents regions of the brain that activate during directed activities, and this network is believed to be involved directly in cognitive function. The TPN consists of the dorsolateral prefrontal cortex, the premotor cortex, and regions of the parietal cortex. Previous studies have found increased activation in TPN regions to be associated with improved performance on cognitive tasks [1-3]. The Task Negative Network (TNN) represents regions that deactivate during task performance. The TNN consists of regions of the prefrontal cortex, posterior cingulate cortex, the hippocampal formation, and regions of the parietal lobule and temporal cortex. The TNN is believed to be involved in the functioning of types of processing that represent the converse of task-related paradigms, such as selfreferential processes including memory formation, the integration of the past and the present, mind-wandering, planning, and decisionmaking. Studies show that decreased activity in the TNN during tasks is associated with improved cognitive performance $[4,5]$. In the absence

Correspondence to: Sarah Khasawinah, Department of Mental Health, Bloomberg School of Public Health, Johns Hopkins University, Baltimore, Maryland, USA, Tel: +1-202-540-0325; E-mail: skhasa@jhu.edu

Key words: task positive network, task negative network, fMRI, older adults, memory, executive functions, cognitive aging

Received: May 06, 2017; Accepted: May 29, 2017; Published: May 31, 2017 
of a task, these regions are active, and the correlations between TNN regions are high, demonstrating that these regions form an integrated functional network [6].

Several regions of the brain implicated in memory and executive functioning overlap with both regions of the TNN and TPN. Therefore, establishing the link between TNN-TPN connectivity and executive functions will help to better provide an integrated understanding of how this domain operates. While stronger connectivity within each of the TPN and TNN has been associated with better executive functions and working memory, the studies investigating the interactions between the two networks are limited and have produced discordant results [2,7-9]. While some studies report interactions between the TPN and TNN in cognitive outcomes, others do not report such results [10-12]. Nevertheless, regional activation studies have found that the TPN activation and TNN deactivation during tasks is associated with better cognitive performance. These connectivity results are also linked to cognition [13]. In resting state fMRI studies, in the absence of a task, studies have shown that the loss of negative correlations between the TNN and TPN is associated with cognitive aging [14].

We investigated the cross-sectional association between brain network connectivity and domains of cognition among aging adults in the Brain Health Study (BHS), an imaging study nested within the Baltimore Experience Corps Trial (BECT) [15-17]. BHS participants were healthy community-dwelling older adults at elevated sociodemographic risk for dementia. We evaluated their performance in the absence of dementia in order to investigate heterogeneity in brainbehavior relationships among at risk older adults. The participants were administered the Sternberg Task, a test of working memory in the fMRI scanner. From this task, we extracted the task negative and task positive networks, assessed the connectivity between each network, and then examined the associations between measures of functional connectivity and various cognitive outcomes related to dementia risk.

We hypothesized that stronger negative correlations between the TNN and TPN, indicative of good interconnection, would be associated with better cognitive performance on tests of memory and executive functions. In other words, stronger negative correlations will indicate that the two networks are more coupled, suggesting that the TPN is activated only during the task, and the TNN is activated only in the absence of the task. The association between cognition and internetwork connectivity may depend on how the networks and cognitive domains are defined.

\section{Materials and methods}

\section{Study sample}

The data were drawn from the BHS, a study nested within BECT, a randomized controlled trial of the Experience Corps (EC) program, a model of senior service in elementary schools. Details about EC and study participants are included elsewhere [17]. Eligibility criteria required at least a $6^{\text {th }}$ grade reading level, and a score on the MiniMental State Exam greater than 23, indicating normal cognition [18]. The study was approved by the Johns Hopkins Institutional Review Board and all participants provided written, informed consent.

\section{fMRI paradigm description}

In this study we employed a one-back version of the Sternberg Task, an event-related paradigm [19]. On each trial, participants viewed a set of four uppercase letters followed by a lowercase letter probe and indicated whether or not that letter had been viewed in the preceding sequence. In the match condition, participants pressed the button in the right hand if the letter was present in the preceding sequence; in the non-match condition, participants pressed the button in the left hand if the letter was not present in the preceding sequence. Each participant completed a total of 40 trials, consisting of 20 trials of the match condition and 20 trials of the non-match condition. Figure 1 outlines an example of a match and non-match trial.

The experimental design was jittered and each inter stimulus interval (ISI) varied from 1.5 to 18 seconds. To investigate activity in the task negative and task positive networks, fMRI event data obtained during both ISI's and task processing were examined.

\section{Data acquisition}

All imaging was performed on a 3T Intera Phillips scanner (Best, the Netherlands). Functional data were collected using $\mathrm{T} 2^{*}$-weighted spin-echo, echo-planar sequence sensitive to detect the blood oxygen level contrast (repetition time $=1500 \mathrm{~ms}$; echo time $=30 \mathrm{~ms}$; slice thickness $=4 \mathrm{~mm} / 1 \mathrm{~mm}$ gap; 30 slices, interleaves acquisition; flip angle $=70$ degrees; matrix $=64 \times 64$; field of view $=240 \mathrm{~mm}$ ). Whole brain coverage was obtained with 30 interleaved slices. A total of 480 volumes were acquired for each participant in a single run.

A structural image was also collected for each participant using the magnetization prepared rapid-acquisition gradient echo protocol (repetition time $=8 \mathrm{~m}$, echo time $=3.6 \mathrm{~ms}$, field of view $=256 \mathrm{~mm}$, matrix $=256 \times 256$, slice thickness $=1 \mathrm{~mm} ; 200$ slices).

\section{Preprocessing of functional data}

All fMRI data processing, unless otherwise noted, was carried out using SPM8 (http://www.fil.ion.ucl.ac.uk/spm). Preprocessing of the functional data included slice-time correction to account for the different times that each volume was acquired during a sequence. Rigid body transformations were applied to correct for movement of the head in the scanner. Each functional scan was then co-registered to its corresponding structural image, and normalized spatially into standard space, using the Montreal Neurological Institute (MNI) image. Lastly, spatial smoothing was conducted using a $7.0 \mathrm{~mm}$ full width at half maximum (FWHM) Gaussian kernel, and high-pass temporal filtering.

Additionally, preprocessing of the structural data entailed removal of non-brain structures using the brain extraction tool in FMRIB's (Oxford Center for Functional MRI of the Brain) software library (FSL) 4.1.9 (www.fmrib.ox.ac.uk/fsl) [20,21]. Functional data were overlaid on the MNI template.

The final sample consisted of 85 participants who had both usable fMRI data and complete cognitive tests obtained outside of the scanner.

\section{Brain networks}

After preprocessing the data, we extracted each brain network

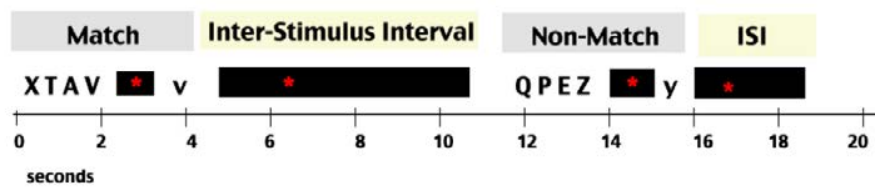

Figure 1. The Sternberg Paradigm. This figure shows a snapshot of the Sternberg Task, as participants experienced it in the scanner. The task periods, match and non-match, are indicated in light gray; the non-task periods, the inter-stimulus intervals, are indicated in light yellow. The time of each period is diagrammed along the horizontal axis in seconds. Each stimulus letter sequence was presented for 2 seconds followed by an average of 3 seconds of a central fixation cross. 
by utilizing regions of interest (ROI) across the entire time sequence of the scan. We utilized a priori regions of $6 \mathrm{~mm}$ spheres based on a landmark fMRI study of 1,000 young healthy adults [22]. For the TPN, we used the following: (A). Anterior control network; (B). Medial control network; (C). Lateral control network; (D). Dorsal attention network; (E). Premotor cortex; and (F). Superior parietal cortex. For the TNN, we used: (A). Prefrontal cortex (PFC); (B). Inferior parietal lobule (IPL); (C). Lateral temporal cortex (LTC); (D). Dorsal medial prefrontal cortex (dMFC); (E). Parahippocampal cortex (PHC); Posterior cingulate/retrospenial cortex (PCC/Rsp). The locations for these regions are shown in Appendices $\mathrm{A}$ and $\mathrm{B}$. These regions have been previously shown to intrinsically belong to the TPN and TNN respectively, and by extracting the BOLD signals across the entire sequence of the task, we anticipate that the TPN network will activate during the trials and TNN network will activate in the brief ISI periods between trials.

The procedure for calculating brain connectivity was to extract the BOLD signal across the entire time sequence for each ROI. Each network consists of a set of time courses. We computed the correlation coefficients between each of the subcomponent pairs both within and between the TNN and TPN for each subject. This resulted in an $\mathrm{p} \mathrm{x}$ $\mathrm{p}$ connectivity matrix for each of the subjects, where $\mathrm{p}$ represents the number of sub-networks. Then we took the average of the matrices across all participants' networks, and plotted the resulting average connectivity matrix.

The signal included both data obtained during ISI's and task processing. The TPN regions elicited stronger signal during task processing, while the TNN regions elicited stronger signals during ISI's.

The connectivity matrix contains the average correlation coefficients for each sub-network pair, with possible values ranging from -1 to 1 . Values close to 0 indicate that the two sub-networks exhibit no relation. Positive values indicate that the two sub-networks operate in tandem such that the activation of one network is associated with the activation of the other network. Negative values indicate that the two sub-networks operate in opposition such that the activation of one network is associated with the deactivation of the other network. We expected that intra-network, the sub-networks will exhibit positive correlations; and inter-network, the sub-networks will exhibit negative, or, anti-correlations [23-26].

\section{Cognitive measures}

Study participants completed tests of executive function and memory, both of which decline with age and predict the onset of dementia [27-31].

To assess executive functions, we administered two tests outside of the scanner: the Trail Making Test (TMT), and the Digit Span Test (DST). The TMT is a visuomotor task of set shifting consisting of two parts, $\mathrm{A}$ and $\mathrm{B}$, to measure psychomotor speed and task switching, respectively. Details are outlined in Carlson, et al. 2009 [32]. Part A is used as an adjustment for speed. Performing Part B requires working memory, mental flexibility, attention, task switching, and rapid visual processing [2]. To be consistent with scoring for other cognitive tasks given, the TMT times were multiplied by negative one so that a higher score would indicate better cognitive performance. The DST is a task of working memory and also consists of two parts, Forward and Backward. DST Backward requires that one recall ascending sequences of numbers in reverse order, which requires working memory [33,34]. A higher score on DST Backward indicates better performance. We created a summary Executive Function (EF) score from the TMT, Part-B and DST Backwards by standardizing these scores, converting them into z-scores and summing them.

To assess episodic memory, we employed the Rey Auditory Verbal Learning Test (RAVLT) [33,35]. Participants were read a list of 15 words, and were asked to remember as many as possible over a sequence of five learning trials. We averaged the normalized scores on the five trials of RAVLT.

\section{Statistical methods}

Linear models were used to assess the association between inter TNN-TPN connectivity and the cognitive outcomes EF and Memory. Both models also included sex, education, and age, given their known associations with cognitive aging [36].

\section{Results}

Table 1 summarizes the demographic and cognitive characteristics of the study sample. The sample is representative of the urban population of older adults in Baltimore: over 90\% are AfricanAmerican, and almost $70 \%$ are female. The average education level represents two years of post-secondary schooling.

\section{Cognition}

Unadjusted raw aggregated and normalized cognitive data are summarized in Appendi x C, Table 2. Figure 4 exhibits the distributions of these scores, which reflect a normal distribution. For EF, the distributions of men and women were similar, as indicated in pink and purple, respectively. However, for memory, men's performance was poorer than that of women.

\section{Inter-regional functional connectivity}

Before showing the association between brain network connectivty and cognitive outcomes, we first illustrate the inter-regional functional connectivity in the BOLD signals. Figure 2 is a plot of the time courses for each of the sub-networks for the 85 participants. The participants are stacked horizontally along the $\mathrm{x}$-axis, and the BOLD signals are indicated on the $y$-axis. While the plot reflects the variable nature of the raw data, some patterns are visible. For instance, the dorsal attention

Table 1. Brain-Behavior Study Sample $(\mathrm{N}=85)$.

\begin{tabular}{|c|c|}
\hline Characteristic & Mean \\
\hline Age, in years (SD) & $67.44(6.2)$ \\
\hline Male, $\mathrm{n}(\%)$ & $23(27.1)$ \\
\hline African American, $\mathrm{n}(\%)$ & $79(92.9)$ \\
\hline Education, in years (SD) & $14.03(2.75)$ \\
\hline MMSE $^{1}$ (SD) & $28.46(1.35)$ \\
\hline Geriatric Depression Scale (SD) & $1.02(1.8)$ \\
\hline
\end{tabular}

${ }^{1}$ MMSE is the Mini-Mental State Examination.

Table 2. The Effect of Functional Connectivity on Memory.

\begin{tabular}{|c|c|c|c|c|}
\hline Variable & Meaning/Unit ${ }^{1}$ & $\begin{array}{c}\text { Coefficient } \\
\text { Estimate }\end{array}$ & $\begin{array}{c}\text { Standard } \\
\text { Error }\end{array}$ & P-Value \\
\hline Education & Years & 0.1 & 0.04 & 0.03 \\
\hline V24 & C-B, D-E & 1.05 & 0.51 & 0.05 \\
\hline V25 & C-B, D-F & 1.73 & 0.89 & 0.05 \\
\hline V36 & C-C, D-E & -0.92 & 0.46 & 0.05 \\
\hline V57 & C-E, D-B & -1.59 & 0.61 & 0.01 \\
\hline \multirow[t]{2}{*}{ V71 } & C-F, D-D & -1.8 & 0.77 & 0.02 \\
\hline & & Model Fit & R-squared & 0.61 \\
\hline
\end{tabular}

${ }^{1} \mathrm{C}$ stands for Control Network. D stands for Default Network. The graphical displays and descriptions of these networks are contained in Appendices A and D, respectively. 


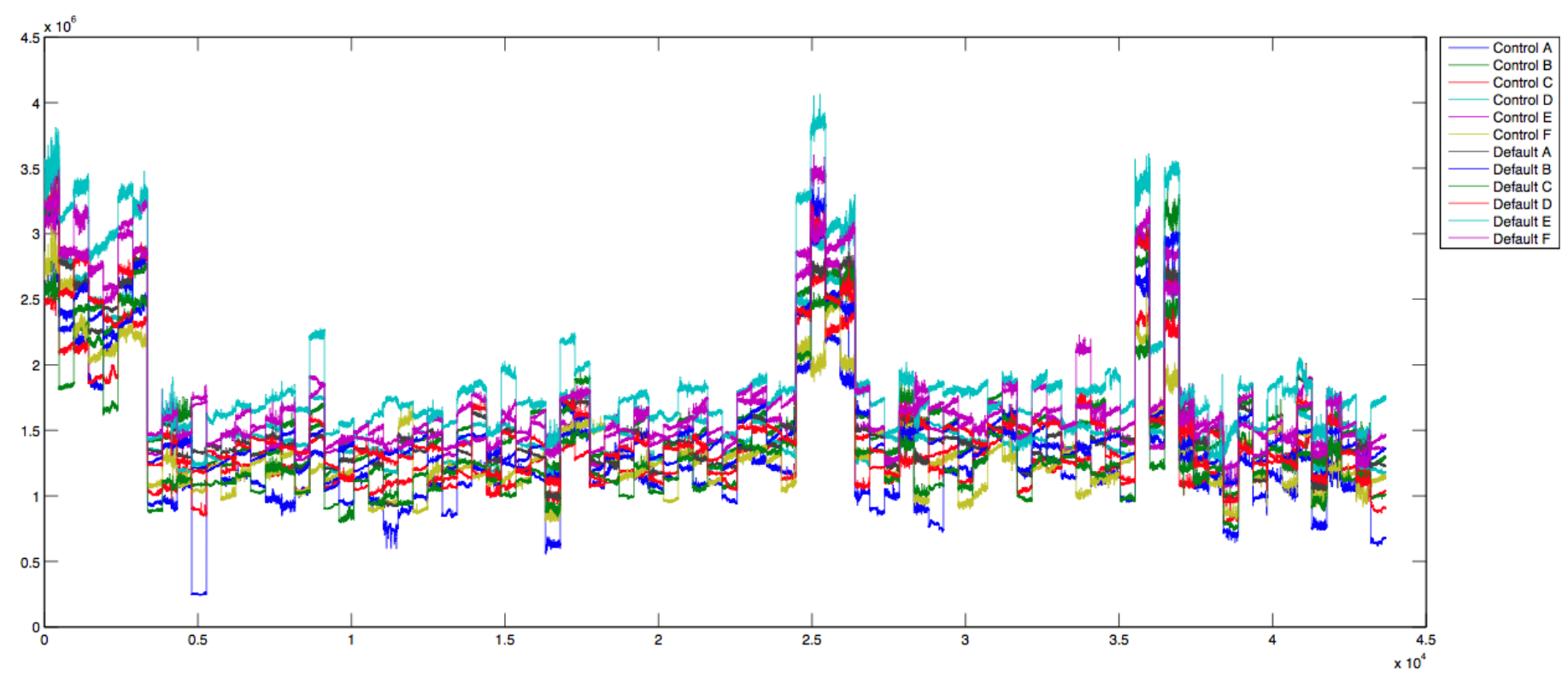

Figure 2. This figure shows the raw time courses for each of the ROI-based sub-networks for the 85 participants. The $\mathrm{x}$-axis represents the participants, which are concatenated side by side; and the $y$-axis represents the fMRI BOLD signals. The legend on the right indicates how the time course are color coded according to the sub-network including Control A - F and Default A-F, representing the TPN and TNN respectively.

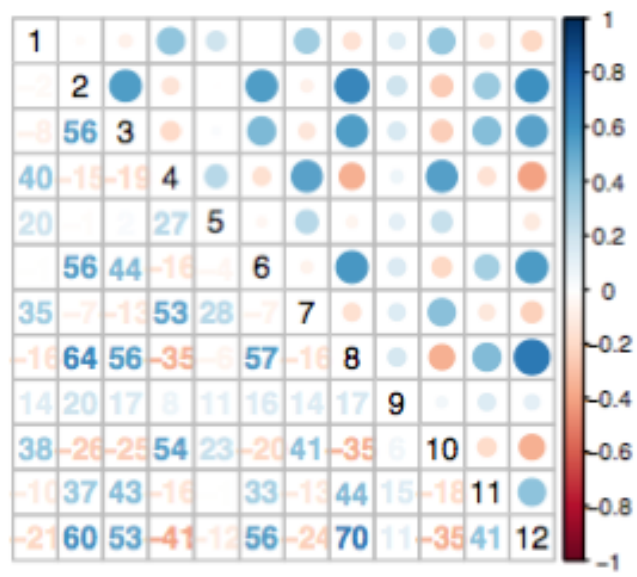

1. Anterior control network
2. Medial control network
3. Lateral control network
4. Dorsal attention network
5. Premotor cortex
6. Superior parietal cortex
7. prefrontal cortex (PFC)
8. Inferior parietal lobule (IPL)
9. Lateral temporal cortex (LTC)
10. dorsal medial prefrontal cortex (dMFC)
11. parahippocampal cortex (PHC)
12. posterior cingulate/retrospenial cortex
(PCC/Rsp)

Figure 3. This connectivity matrix shows the average correlation values across the 85 study participants. Sub-networks 1-6 represent the TNN and sub-networks 7-12 represent the TPN, as indicated in the legend on the right. Rows 1-6 crossed with columns 1-6 represent the intra-task positive network. Rows $7-12$ crossed with columns 1-7 represent the inter TNN-TPN network. Rows 7-12 crossed with columns 7-12 represent the intra-task negative network. Shades of red indicate positive correlations and shades of blue indicate negative correlations.

network (Control Network D), illustrated in teal-blue, and consistently shows a BOLD signal higher than any of the other sub-networks. Similarly, the inferior parietal lobule network (Default Mode Network B), illustrated in royal-blue consistently shows a BOLD signal lower than any of the other sub networks. This pattern suggests that these two networks should be anti-correlated, a finding supported by a negative correlation between the TNN and TPN.

These illustrations of raw time courses provide an indication of the connectivity between the networks; and, to quantify and visualize the connectivity more clearly, we compute the correlation between each network pair. Figure 3 exhibits the average correlation values across all study participants. Red indicates positive correlations and blue indicates negative correlations, also known as anti-correlations. The connectivity matrix is equivalent along the diagonal, and the upper triangle exhibits a visualization of the correlation coefficient values, while the lower triangle exhibits the precise coefficient values. In the upper triangle, the diameter of the circle represents the amount of correlation.
Figure 3 shows that there are substantial anti-correlations between the TNN and TPN. In particular, networks 4 and 8, the dorsal attention network and the inferior parietal lobule network, which are also notable in the plot of the raw time courses in Figure 2, are indeed anticorrelated. Networks 4 and 12 (dorsal attention network and posterior cingulate/retrospenial cortex (PCC/Rsp) network) are also substantially negatively correlated. These anti-correlations provide support for the hypothesis that the TNN and TPN are decoupled. However, there are also strong positive correlations between the networks. Networks 2 and 8 (medial control network and inferior parietal lobule (IPL) network); 2 and 12 (medial control network and PCC/Rsp network); 3 and 8 (lateral control network and IPL); and 3 and 12 (lateral control network and PCC/Rsp network) are each positively correlated with each other.

\section{Brain network connectivity-cognition}

Linear regression was used to link brain connectivity with cognitive outcomes. There were a total of six TNN sub-networks and six TPN 
(a)

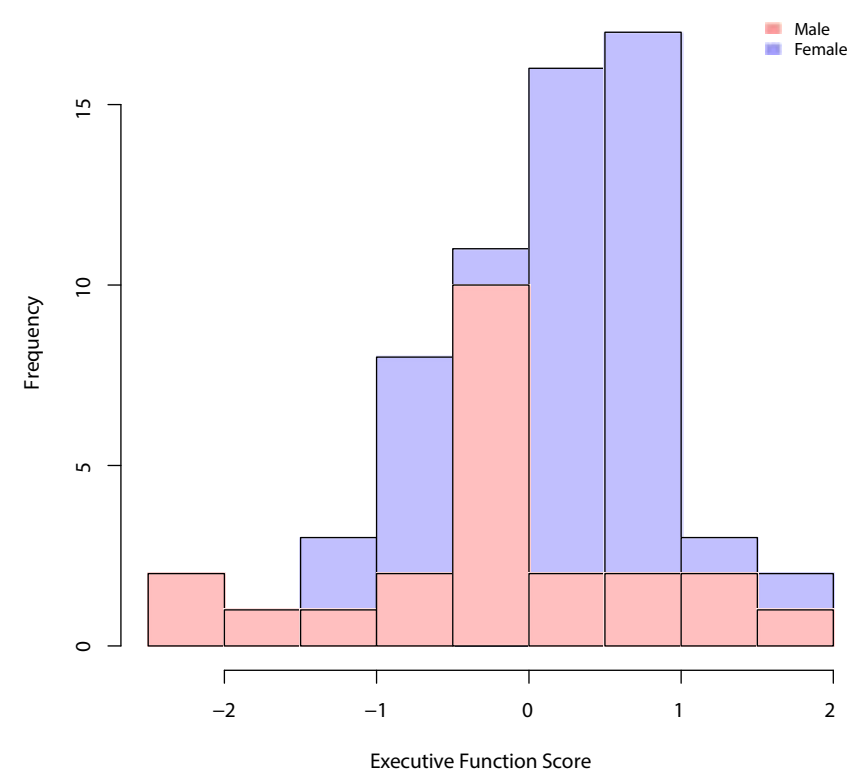

(b)

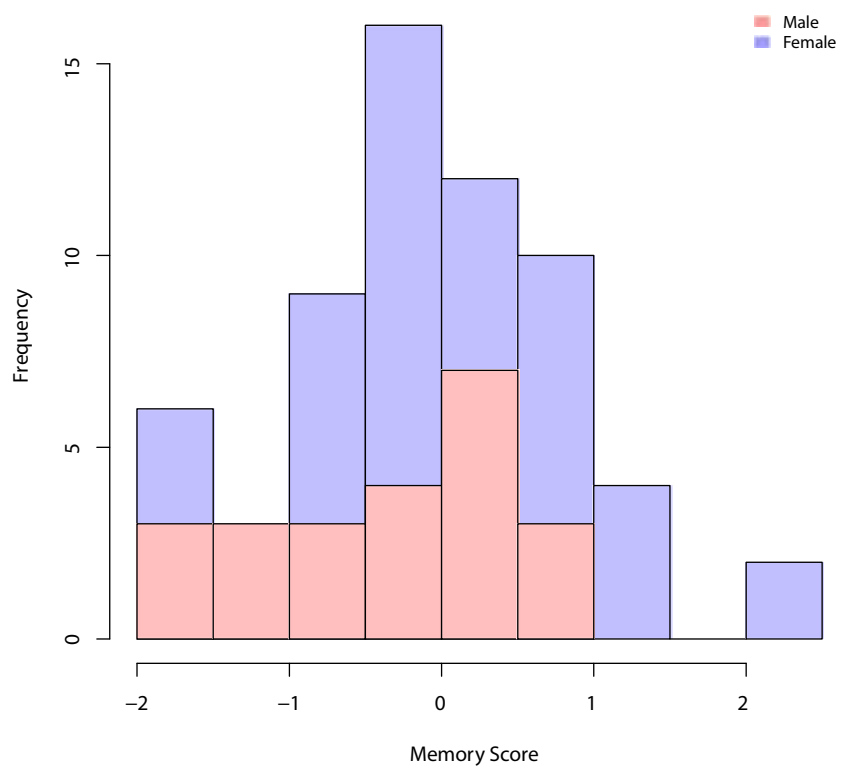

Figure 4. Histograms showing the distributions of the cognitive scores in men (pink) and women (purple). (a) Distribution of Executive Function score; (b) Distribution of Memory score.

sub-networks, which yielded $12 \times 12$, or 144 intra and inter-network correlation pairs. Since the connectivity matrix is symmetric along the diagonal, and each sub-network is perfectly connected with itself, this left 66 unique correlation pairs of interest: 15 representing connectivity within the TPN, 15 representing connectivity within the TNN, and 36 representing inter-network connectivity.

Prior to conducting the linear models to assess the association between brain network connectivity and cognitive outcomes, we performed an exploratory analysis. We computed the correlation between each pair of sub-networks' correlation coefficients with each cognitve outcome, EF and memory, stratified by sex. These correlations are illustrated in Figure 3. Larger circles indicate larger correlation values. Smaller circles indicate smaller values. The correlation values range from -1 to 1 , and in color from red to blue, with white representing 0. For females for both EF and memory, the values representing the association between brain network connectivity and cognitive outcomes hover closer to zero, than for males. The cells with large circles indicate that sub-network pair has a stronger association with a given cogntitive outcome.

Executive function vs. memory: In the Memory Model, shown in Figure 5(a), we included all 36 TPN-TNN sub-network correlation pairs to investigate the relation between inter TPN-TNN connectivity and the Memory score. These sub-network correlation pairs will be referred to as connectivity scores. The graph of the coefficient estimates for the connectivity scores illustrates that 20 out of 36 of the coefficients are negative-these connectivity scores were inversely associated with the memory score. Figure 5 also includes 95\% confidence intervals for the coefficient estimates, which demonstrate a large amount of variability in the data. The statistically significant coefficients at the P-vale of 0.05 are indicated with an asterisk in Figure 5. The significant
Table 3. The Effect of Functional Connectivity on Executive Function.

\begin{tabular}{|c|c|c|c|c|}
\hline Variable & Meaning/Unit $^{\mathbf{1}}$ & $\begin{array}{c}\text { Coefficient } \\
\text { Estimate }\end{array}$ & $\begin{array}{c}\text { Standard } \\
\text { Error }\end{array}$ & P-Value \\
\hline Education & Years & 0.94 & 0.05 & 0.05 \\
\hline V57 & C-E, D-B & -1.95 & 0.67 & 0.005 \\
\hline & & Model Fit & R-squared & 0.47 \\
\hline
\end{tabular}

${ }^{1} \mathrm{C}$ stands for Control Network. D stands for Default Network. The graphical displays and descriptions of these networks are contained in Appendices A and D, respectively.

findings are highlighted in Table 3. Five connectivity scores were significant, three of which were negatively associated with the memory score. Two of these significant sub-networks were highly integrated with corresponding sub-networks from the TNN (Control B) and TPN (Default E), respectively.

In the Executive Function Model, shown in Figure 5(b), we investigated the relation between inter TPN-TNN connectivity and the EF score. The figure shows that 23 connectivity scores have negative coefficient estimates, indicated by an inverse relation with the executive function score. However, the $95 \%$ confidence intervals are very wide, and only one connectivity score is significant at the 0.05 level, as indicated in Table 3. This connectivity score represents the correlation between subnetwork Control E and Default B, and has a coefficient estimate of -1.95 . This connectivity score also turned out to be significant in Model 1.

\section{Men vs. women}

In the brain-behavior models, sex was included as a covariate, and being male was associated negatively with the Executive Function and Memory scores. An exploratory analysis revealed different patterns for men and women. Figure 6 provides an example in which sex modifies the relation between brain connectivity and cognitive outcomes. For 


\section{A. Executive Function in Females}

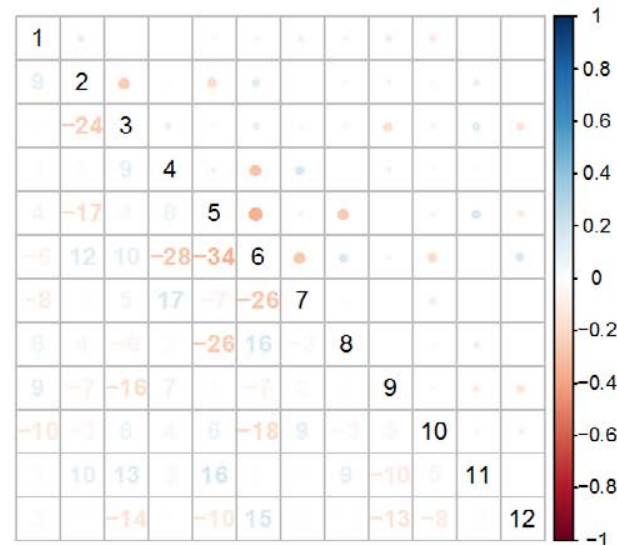

C. Memory in Females

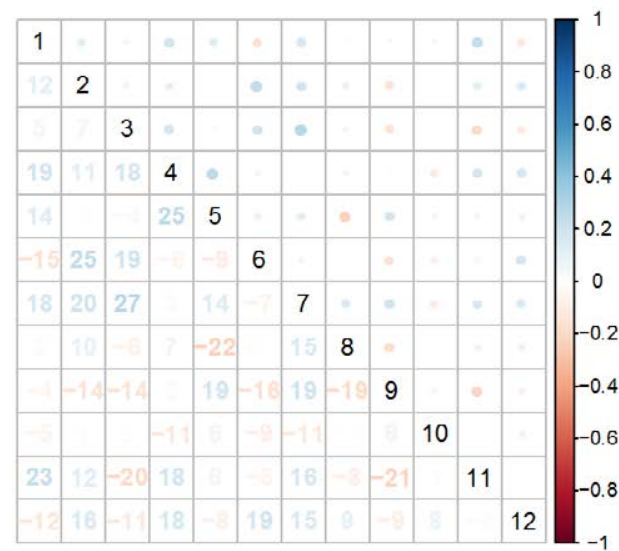

B. Executive Function in Males

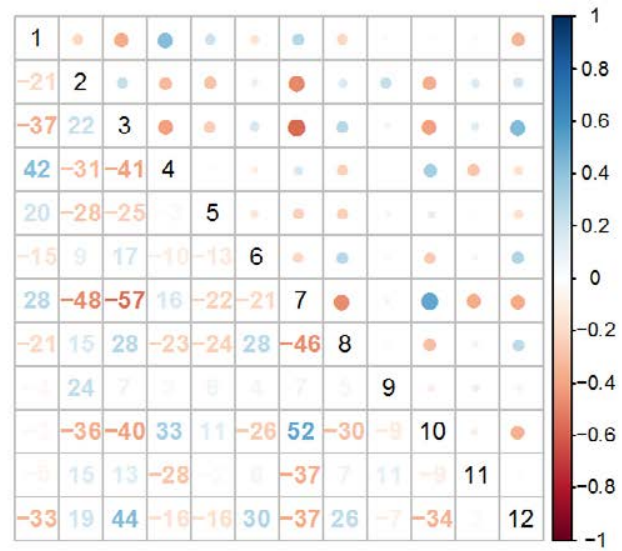

\section{Memory in Males}

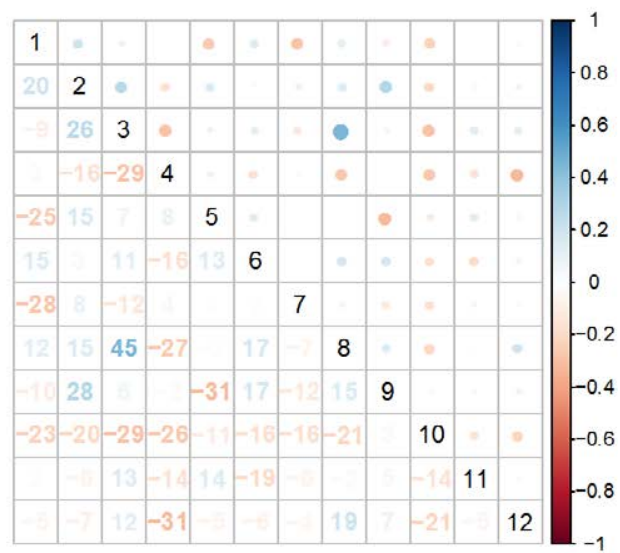

Figure 5. Exploratory Analysis of Brain-Behavior Associations. The numbers represent the sub-networks as follows: (1). Control A; (2). Control B; (3). Control C; (4). Control D; (5). Control E; (6). Control F; (7). Default A; (8). Default B; (9). Default C; (10). Default D; (11). Default E; (12). Default F; This set of matrices shows the average correlations between the functional connectivity score and cognitive outcomes for each sub-network. The numbers in the lower triangles represent the correlations. The upper row, panels A and B, shows the average correlations for the executive functions outcome for women and men respectively. The lower row, panels C and D, shows the average correlations of functional connectivity with memory scores for women and men respectively.

the inter-network correlations displayed in Figure 6, women, in red, exhibited the expected pattern: correlations between the TNN and TPN were inversely associated with cognitive scores; and men, in blue, exhibited the opposite: inter-network correlations were positively associated with cognitive scores.

\section{Discussion}

In this cross-sectional study investigating the associations between brain connectivity and cognition in a group of non-demented older adults, we found that the association of brain connectivity and cognition differed for Executive Function (EF) and Memory. EF was negatively associated with inter-network connectivity while Memory was variably associated with inter-network connectivity, and sex modified this relationship.

The lack of strong anti-correlations between the TNN and TPN suggests that these two networks were not fully decoupled in our sample of socio-demographically diverse community-based older adults. The majority of findings in the literature with conclusions to the contrary are based on studies of young healthy adults. Therefore, it may be that while the TNN and TPN are decoupled in young healthy adults, the two networks become less decoupled during aging. In fact, other studies in children and older adults are also finding that the relation between the TNN and TPN may be more complicated than the common binary understanding. Fair et al. found that in children ages 7-9 years old, the TNN exhibits only sparse correlations with itself [37]. Developmental models of neuroanatomy demonstrate that the last areas to develop are also the first to deteriorate, which suggests that functional connectivity in the brains of children may provide a model helpful for the understanding of brain networks in older adults [37]. Also, Steffener et al. found that in older adults, the TNN remains active during task performance, and they hypothesize that the functioning of this network, in parallel with the TPN may actually serve to enhance performance for older adults [38]. The speculation that the TNN and TPN operating together may enhance performance suggests that for older adults, these networks may function in a compensatory mechanism. Perhaps the binary organization of the brain exhibited in young healthy adults is not optimal for older adults, and in fact the coupling of the two networks is a natural part of cognitive aging.

\section{Executive function $v s$. memory}

Both models fit the data well, indicating that the connectivity between the TNN and TPN may function together to modulate 


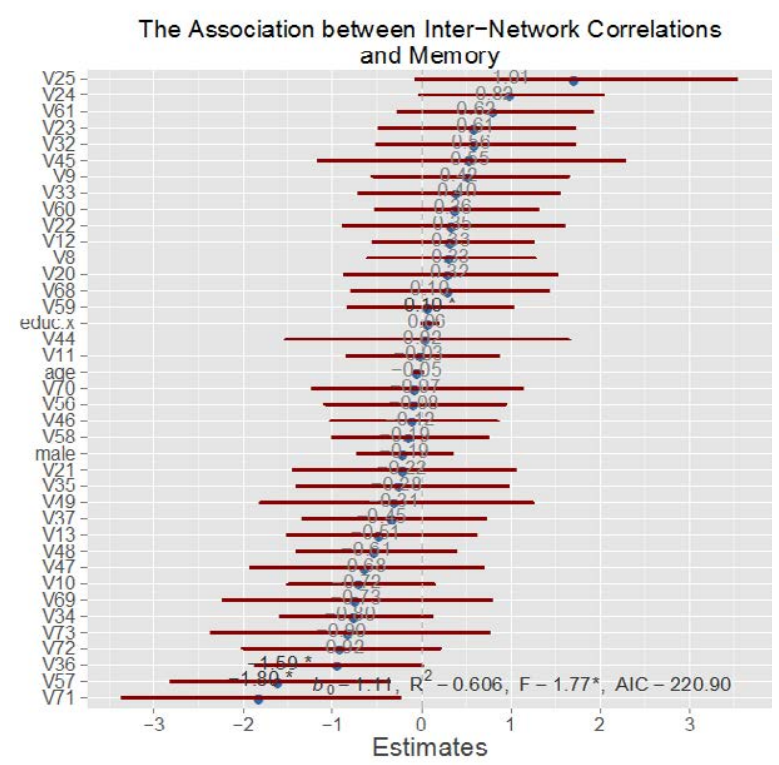

(a)

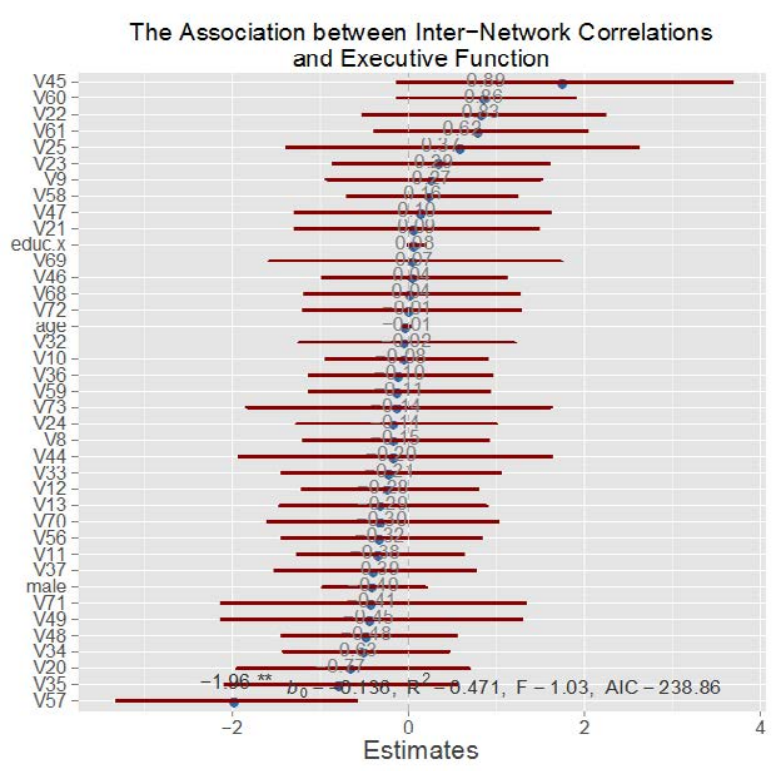

(b)

Figure 6. Overview of Inter-Network Connectivity Models. These plots show the results of the inter-network connectivity models for (a) memory and (b) executive function. The coefficient estimates for each of the inter-network connectivity pairs is plotted together with its $95 \%$ confidence interval. Covariates that are significant at the 0.05 level are indicated with an asterisk. The meanings of the connectivity pairs, V20 ... V73 are contained in Appendix D. In these models, males and females are pooled together.
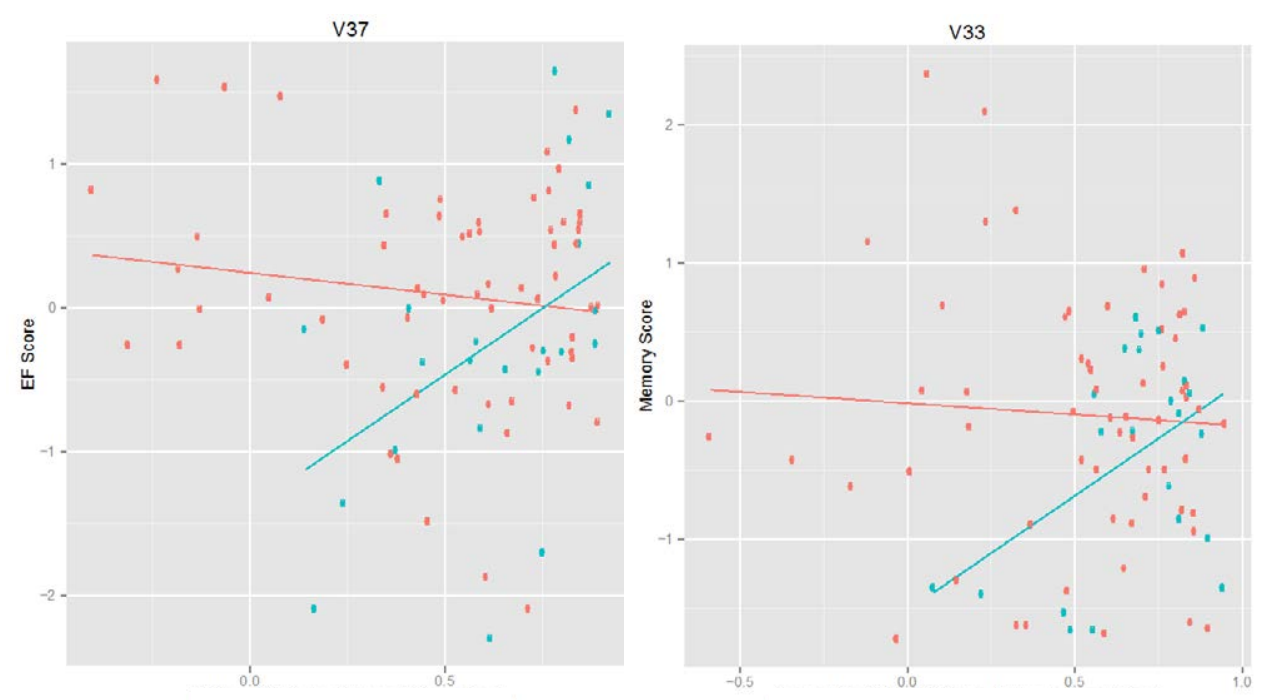

(a) Inter-Network Correlations

(b) Inter-Network Correlations

Figure 7. The Association of Sex with Brain-Behavior Outcomes. This figure shows the relation between inter-network correlation and cognitive performance, color coded by sex with women in red and men in blue. The x-axis represents the connectivity scores and the y-axis represents the cognitive performance scores. Panel (a) shows the association between $\mathrm{V} 37$ (Control C v. Default F) connectivity scores and executive function. Panel (b) shows the association between V33 (Control C v. Default B) connectivity scores and memory.

cognitive performance. However, the strengths of association did not uniformly fit the directions hypothesized. While we hypothesized that inter-network connectivity would be negatively correlated with cognitive performance, the results support this hypothesis for selected sub-network pairs, and show that in general, both positive and negative associations exist. This variability can be explained by the emerging literature that functional connectivity is dynamic, and may change over time, and can be influenced by a number of daily (i.e., sleep), lifestyle (i.e., physical activity), or other proximal factors (i.e., previous cognitive demands). Lastly, the Memory model fit the data better than the Executive Function model ( $\mathrm{R}$ squared 0.61 vs. 0.47), suggesting that coupling between the TNN and TPN was more important in the modulation of Memory than EF. However, it is worth noting that in our study, the Memory and EF scores were derived differently. The Memory score was derived using the aggregate of five trials of a single test while the EF score was derived using the aggregate of two tests. In the fMRI scanner, the participants completed the Sternberg paradigm, a working memory test which includes components of both memory and EF, which could have also influenced these results. 
In the Memory Model, five connectivity scores achieved statistical significance, with both positive and negative associations. Specifically, Control B, located in the anterior portion of the TPN, was positively associated with Default E and Default F, which are located in the posterior portions of the TNN, at the parahippocampal cortex (PHC) and posterior cingulate cortex (PCC), respectively. The positive association between these TPN-TNN network pairs and memory suggest that the anterior regions of the TPN are communicating with the posterior regions of the TNN in memory function. This result supports findings from the literature, as the PCC has been implicated in memory retrieval, and known to be one of the most highly connected regions of the brain, representing a node of the TNN [39]. The negative connectivity scores support the hypothesis that greater inverse coupling between the networks is associated with better cognitive performance. These connectivity scores represented sub-networks located in multiple locations in the brain, and replicate results from the literature that have shown that better memory performance is associated with negative correlations between the TNN and TPN [1].

In the Executive Function Model, only one connectivity score achieved statistical significance: the sub-network pair Control E, in the medial portion of the TPN, and Default $\mathrm{B}$, in the inferior parietal lobule, was inversely related to the EF score. This result is consistent with the current state of the literature. Kelly et al. found that the anticorrelation between task-positive and task-negative networks was inversely associated with response time, a component of EF [40]. Thus, these findings support the inverse coupling hypothesis between the TNN and TPN, as a mechanism for cognitive health.

\section{Men vs. women}

A consistent finding in the neuroimaging literature has been that differences exist in brain connectivity of men and women [41,42]. Demographic and health differences between men and women together with sex differences in brain morphology and differential risk for age-related neuropathologies justify this a priori stratification. We found that in women, inter-network connectivity is inversely associated with cognition, while men exhibited the opposite. These findings in men suggest a different mechanism was operating. Since the men consistently scored lower than women on the cognitive tests, perhaps the differences in connectivity patterns represent a biological explanation for these behavioral differences. However, differential sample size between men and women could also be responsible, in part, for the different pattern of results. These results revealing different qualitative patterns for men and women are determined from exploratory analysis, and a future direction would be to conduct formal analyses stratified by sex. Longitudinal change models would allow us to evaluate whether the reverse connectivity patterns in males precedes performance on the Executive Function and Memory tests.

\section{Strengths and limitations}

There are a number of strengths of this study that warrant mention. One strength arises from the study design. Because the data came from an event-based fMRI trial design with a cognitive task rather than resting state, the differences between the TNN and TPN can be measured within the same protocol and relative differences may be easier to identify. Larger differences between the networks, engendered by the task, would create larger correlations and anti-correlations, which would exceed the spontaneous correlations, due to physiological noise present in fMRI, and make it easier to detect true signals. By examining these differences within the same event-based task, we controlled for measurement related confounds, such as fatigue and motion that may occur when comparing two different fMRI sequences.

Also by design, the study population consists of a community based sample that reflected the aging population of Baltimore City, including minorities and a variety of socioeconomic and educational backgrounds. The majority of the sample is African American and female, which addresses the need for more studies among underrepresented minorities at elevated risk for health disparities. We included only older adults to follow the precedent in the resting state fMRI literature of identifying methods in aging adults that will help further elucidate the neural mechanisms related to modification of risk for dementia [43-45].

A third strength is in the relation of brain network connectivity with behavior. This investigation considered not only mechanisms in the brain, but also their relation to behavior, outside of the scanner environment. Further, these results contribute to the development of standardizable tools to detect pre-clinical changes in cognition to help predict dementia pathology while there is still time to intervene.

The event-related study design can also be viewed as a limitation. Brain network analyses are traditionally performed on resting state data, and therefore, it would be helpful to replicate some of these analyses using resting state fMRI data. Nevertheless, because the TNN and TPN are intrinsic to the organization of the brain, studies support the inherent existence of these networks, and sensitivity to detecting each, both in resting state data and during cognitive processing tasks [6]. The validity of using data from event-related study designs compared to task-free protocols has also been previously studied. In 2007, Fair et al. concluded that interleaved resting-state data (such as that from blocked event related fMRI designs) yielded resting state connectivity patterns that were both qualitatively and quantitatively similar to continuous rest data [23]. The similarities in findings across study protocols adds further support to the intrinsic existence of the networks, and suggests that for a more nuanced understanding of the relationship between networks task-based fMRI, rather than task-free designs ought to be utilized. It would be valuable to perform parallel analyses on other event-related paradigms to determine the extent to which these networks are driven by the demands of the task.

Thus, while the literature is based on resting state fMRI, we propose that if these networks exist spontaneously, then their presence ought to be magnified in the presence of a task. Therefore, using data from an event-based paradigm may represent strength rather than a limitation. The consistency in findings across both event-based and resting state fMRI studies provides further support for the underlying biology of the relation between brain networks, and suggests that one day; this tool can be developed as a robust measure of brain health.

Additionally, while 85 participants constitute a large study sample for fMRI brain imaging, the linear regressions with 35 variables may have led to collinearity and potential for Type I error. However, this potential is mitigated by our study's replication of patterns observed in other studies.

Lastly, the positive and negative correlations observed warrant further investigation. Perhaps these positive and negative directions represent an artifact of multiplicity due to the large number of correlations studied. Dynamic functional connectivity could also probe at these differences using sliding window correlations, which involves computing correlation coefficients for overlapping successive time periods, rather than simple averages $[46,47]$.

A future direction may be too comprehensively map the brain 
connectivity-behavior relation in older adults would be to use a fully data-driven approach using every possible seed voxel in the brain rather than a priori seeds for the connectivity scores. However, strength of the ROI approach used here is that the selected regions represent constituents of brain networks with known connectivity trends that can be well integrated into the existing literature.

\section{Implications: biomarker development}

This work is conducted in a primarily Black and underserved segment of the aging population at an elevated sociodemographic risk for Alzheimer's disease (AD). Patterns of TPN and TNN activation replicated those observed in other primarily White sample, and has a strong application to broader biomarker development. Brain network connectivity has proven a promising area of research in $\mathrm{AD}$ research. The literature has focused extensively on dysfunction of $\mathrm{TNN}$ and $\mathrm{AD}$ risk. This investigation further probes the association between coupling of the TNN and the TPN, and cognitive outcomes prior to clinical presentation of cognitive impairment. These methods provide a framework for using fMRI imaging to prospectively follow these participants for incident cognitive impairment and dementia to determine whether this dynamic measure may be used in future research to detect pre-clinical changes in brain connectivity as well as intervention-related changes in response to lifestyle modifications, such as Experience Corps.

Recent evidence that brain pathology does not consistently correspond well with clinical symptoms weakens present understanding of $\mathrm{AD}$ etiology $[48,49]$. Another useful application will be to search more closely for markers of aging in the brain, such as those suggested by Jack et al. in his 2013 model laying out the time course for preclinical biomarkers of brain aging such as cerebrospinal fluid (CSF) tau, a protein implicated in maintaining the structure of cells; brain volume and cortical thickness via MRI; and cognitive impairments from neuropsychological tests [50,51]. An fMRI biomarker would be advantageous compared to other imaging technologies because fMRI is minimally invasive and can be easily standardized to measure preclinical changes in cognition, which can help to differentiate between normal aging and $\mathrm{AD}$ pathology.

\section{Acknowledgments}

The authors extend their appreciation and thanks to the study participants for their critical contributions to this research. Recruitment and baseline evaluation was supported by the Johns Hopkins Neurobehavioral Research Unit and a supplement to the National Institute on Aging (BSR grant P01 AG027735-03). S.K.'s research was supported by the Johns Hopkins Sommer Scholarship.

\section{Author contributions}

S.K., M.C.C., and B.C. conceived and designed the analytic plan; K.I.E. and A.F.K. contributed to the study design; S.K. and Y.F.C. preprocessed the data; S.K. performed the analysis; S.K. wrote the paper and all authors edited the paper. All authors reviewed and approved the final manuscript.

\section{Conflicts of interest:}

The authors declare no conflict of interest.

\section{References}

1. Hampson M (2010) Relating Variations in Network Connectivity to Cognitive Function, New Haven.
2. Seeley WW, Menon V, Schatzberg AF, Keller J, Glover GH, et al. (2007) Dissociable intrinsic connectivity networks for salience processing and executive control. $J$ Neurosci 27: 2349-2356. [Crossref]

3. Song M, Zhou Y, Li J, Liu Y, Tian Let al. (2008) Brain spontaneous functional connectivity and intelligence. Neuroimage 41: 1168-1176. [Crossref]

4. Mazoyer B, Zago L, Mellet E, Bricogne S, Etard O, et al. (2001) Cortical networks for working memory and executive functions sustain the conscious resting state in man Brain Res Bull 54: 287-298. [Crossref]

5. Shulman GL, Fiez JA, Corbetta M, Buckner RL, Miezin FM, et al. (1994) Common Blood Flow Changes across Visual Tasks: Decreases in Cerebral Cortex. $J$ Cogn Neurosci 9: 648-663. [Crossref]

6. Greicius MD, Menon V (2004) Default-mode activity during a passive sensory task uncoupled from deactivation but impacting activation. J Cogn Neurosci 16: 1484-1492. [Crossref]

7. Rissman J, Gazzaley A, D'Esposito M (2004) Measuring functional connectivity during distinct stages of a cognitive task. Neuroimage 23: 752-763. [Crossref]

8. Leech R, Kamourieh S, Beckmann CF, Sharp DJ (2011) Fractionating the default mode network: distinct contributions of the ventral and dorsal posterior cingulate cortex to cognitive control. J. Neurosci 31: 3217-3224. [Crossref]

9. Rajah MN, Esposito MD (2005) Region-specific changes in prefrontal function with age: a review of PET and fMRI studies on working and episodic memory. Brain 128 1964-1983. [Crossref]

10. Sridharan D, Levitin DJ, Menon V (2008) A critical role for the right fronto-insular cortex in switching between central-executive and default-mode networks. Proc Natl Acad Sci USA 105: 12569-12574. [Crossref]

11. Onoda K, Ishihara M, Yamaguchi S (2012) Decreased Functional Connectivity by Aging Is Associated with Cognitive Decline Decreased Functional Connectivity by Aging Is Associated with Cognitive Decline. J Cogn Neurosci 2186-2198. [Crossref]

12. Charrouda C, Le Barsa E, Deverduna J, Steffenerg J, Molinoa F, et al. (2016) Working memory performance is related to intrinsic resting state functional connectivity changes in community-dwelling elderly cohort. Neurobiol Learn Mem 132: 57-66. [Crossref]

13. Smith S (2015) Linking cognition to brain connectivity. Nat Neurosci. [Crossref]

14. Ferreira LK, Brocanello Regina AC, Kovacevic N, Morais Martin MG, Santos PP, et al. (2016) Aging Effects on Whole-Brain Functional Connectivity in Adults Free of Cognitive and Psychiatric Disorders. Cereb. Cortex 26: 3851-3865. [Crossref]

15. Carlson MC, Seeman T, Fried LP (2000) Importance of generativity for healthy aging in older women. Aging (Milano) 12: 132-140. [Crossref]

16. Fried LP, Carlson MC, McGill S, Seeman T, Xue QL, et al. (2013) Experience Corps a dual trial to promote the health of older adults and children's academic success Contemp Clin Trials 36: 1-13. [Crossref]

17. Carlson MC, Kuo JH, Chuang YF, Varma V, Harris G, et al. (2015) Impact of the Baltimore Experience Corps Trial on cortical and hippocampal volumes. Alzheimers. Dement 1-9. [Crossref]

18. Folstein MF, Folstein SF, McHugh PR (1975) "Mini-Mental State” A Practical Method for Grading the Cognitive State of Patients for the Clinician. J. Psychiat. Res 12: 189198. [Crossref]

19. Sternberg S (1969) Memory-scanning: mental processes revealed by reaction time experiments. Am Sci 57: 421-457? [Crossref]

20. Smith SM, Jenkinson M, Woolrich MW, Beckmann CF, Behrens TEJ, et al. (2004) Advances in functional and structural MR image analysis and implementation as FSL. Neuroimage 23: 208-219. [Crossref]

21. Smith SM (2002) Fast robust automated brain extraction. Hum Brain Mapp 17: 143155. [Crossref]

22. Yeo BTT, Kriene FM, Sepulcre J, Sabuncu MR, Lashkari D, et al. (2011) The organization of the human cerebral cortex estimated by intrinsic functional connectivity $J$ Neurophysiol 106: 1125-1165. [Crossref]

23. Whitfield-Gabrieli S1, Ford JM (2012) Default mode network activity and connectivity in psychopathology. Annu Rev Clin Psychol 8: 49-76. [Crossref]

24. Fransson, P. How default is the default mode of brain function? Further evidence from intrinsic BOLD signal fluctuations. Neuropsychologia 2006, 44, 2836-45.

25. Raichle ME (2010) Two views of brain function. Trends Cogn Sci 14: 180-190 [Crossref] 
26. Vemuri P, Jones DT, Jack CR (2012) Resting state functional MRI in Alzheimer's disease. Alzheimers. Res. Ther 4: 1-9. [Crossref]

27. Carlson MC, Erickson KI, Kramer AF, Voss MW, Bolea N, et al. (2009) Evidence for neurocognitive plasticity in at-risk older adults: the experience corps program. $J$ Gerontol A Biol Sci Med Sci 64: 1275-82. [Crossref]

28. Albert M, DeKosky S, Dickson D, Dubois B, Feldman H, et al. (2011) The diagnosis of mild cognitive impairment due to Alzheimer's disease: recommendations from the National Institute on Aging- Alzheimer's Association workgroups on diagnostic guidelines for Alzheimer's disease. Alzheimers. Dement 7: 270-279. [Crossref]

29. Mielke MM, Okonkwo OC, Oishi K, Mori S, Tighe S, et al. (2012) Fornix integrity and hippocampal volume predict memory decline and progression to Alzheimer's disease. Alzheimers. Dement 8: 105-113. [Crossref]

30. Gross A L, Parisi JM, Spira AP, Kueider AM, Ko JY, et al. (2013) Memory training interventions for older adults?: A meta-analysis. Aging Ment Heal 16: 722-734.

31. Kueider AM, Parisi JM, Gross AL, Rebok GW (2012) Computerized cognitive training with older adults: a systematic review. PLoS One 7: 1-13. [Crossref]

32. Carlson MC, Erickson KI, Kramer AF, Voss MW, Bolea N, et al. (2009) Evidence for neurocognitive plasticity in at-risk older adults: the experience corps program. $J$ Gerontol A Biol Sci Med Sci 64: 1275-1282. [Crossref]

33. Lezak MD, Howieson DB, Loring DW (2004) Neuropsychological assessment (4 $4^{\text {th }}$ edn), Oxford University Press: New York.

34. Groeger JA, Field D, Hammond SM (1999) Measuring memory span. Int J Psychol 1999 34: 359-363. [Crossref]

35. Spreen O, Strauss E (1991) Controlled oral word association. In A compendium of neuropsychological tests 219-27.

36. Van der Elst W, Van Boxtel M, Van Breukelen GJP, Jolles J (2006) The Letter Digit Substitution Test: Normative Data for 1,858 Healthy Participants Aged $24-81$ from the Maastricht Aging Study (MAAS): Influence of Age, Education, and Sex. J Clin Exp Neuropsychol 28: 998-1009. [Crossref]

37. Fair DA, Cohen AL, Dosenbach NUF, Church JA, Miezin FM, et al. (2008) The maturing architecture of the brain's default network. Proc Natl Acad Sci USA 105: 4028-4032. [Crossref]

38. Steffener J, Habeck CG, Stern Y (2012) Age-related changes in task related functional network connectivity. PLoS One 7: e44421. [Crossref]

39. Nielsen FA, Balslev D, Hansen LK (2005) Mining the posterior cingulate: Segregation between memory and pain components. Neuroimage 27: 520-532. [Crossref]
40. Kelly CA, Uddin LQ, Biswal BB, Castellanos X, Milham MP (2008) Competition between functional brain networks mediates behavioral variability. Neuroimage 39: 527-537. [Crossref]

41. Schmithorst VJ, Holland SK (2007) Sex Differences in the Development of Neuroanatomical Functional Connectivity underlying Intelligence found using Bayesian Connectivity Analysis. Neuroimage 35: 406-419. [Crossref]

42. Kilpatrick LA, Zald DH, Pardo JV, Cahill LF (2006) Sex-related differences in amygdala functional connectivity during resting conditions. Neuroimage 30: 452-461. [Crossref]

43. Buckner RL (2004) Memory and Executive Function Review in Aging and AD Multiple Factors that Cause Decline and Reserve Factors that Compensate. Neuron 44 : 195-208. [Crossref]

44. Buckner RL, Andrews-Hanna JR, Schacter DL (2008) The brain's default network: anatomy, function, and relevance to disease. Ann N Y Acad Sci 1124: 1-38. [Crossref]

45. Voss MW, Prakash RS, Erickson KI, Basak C, Chaddock, L, et al. (2010) Plasticity of brain networks in a randomized intervention trial of exercise training in older adults. Front. Aging Neurosci 2: 1-17. [Crossref]

46. Hindriks R, Adhikari MH, Murayama Y, Ganzetti M, Mantini D, et al. (2016) Can sliding-window correlations reveal dynamic functional connectivity in resting-state fMRI? Neuroimage 127: 242-256. [Crossref]

47. Schulz D, Huston JP (2002) The sliding window correlation procedure for detecting hidden correlations: existence of behavioral subgroups illustrated with aged rats. $J$. Neurosci. Methods 121: 129-137. [Crossref]

48. Sperling RA, Aisen PS, Beckett LA, Bennett DA, Craft S, et al. (2011) Toward defining the preclinical stages of Alzheimer's disease: Recommendations from the National Institute on Aging- Alzheimer's Association workgroups on diagnostic guidelines for Alzheimer's disease. Alzheimers Dement 7: 280-292. [Crossref]

49. Whalley LJ (2002) Brain ageing and dementia: what makes the difference? $B r J$ Psychiatry 181: 369-371. [Crossref]

50. Clifford JR, Knopman DS, Jagust WJ, Petersen RC, Weiner MW, et al. (2013) Tracking pathophysiological processes in Alzheimer's disease: an updated hypothetical model of dynamic biomarkers. Neurology 12: 207-216. [Crossref]

51. Jack CR, Lowe VJ, Weigand SD, Wiste HJ, Senjem ML, et al. (2009) Serial PIB and MRI in normal, mild cognitive impairment and Alzheimer's disease: implications for sequence of pathological events in Alzheimer's disease. Brain 132: 1355-1365. [Crossref]

Copyright: (C2017 Khasawinah S. This is an open-access article distributed under the terms of the Creative Commons Attribution License, which permits unrestricted use, distribution, and reproduction in any medium, provided the original author and source are credited. 